\title{
Does Older Age Lead to Higher Risk for Neutropenia in Patients Treated with Paclitaxel?
}

\author{
Marie-Rose B. S. Crombag ${ }^{1,2}$ (D) - Stijn L. W. Koolen ${ }^{3,4}$ • Sophie Wijngaard ${ }^{3}$ • Markus Joerger ${ }^{5}$ • \\ Thomas P. C. Dorlo ${ }^{1,2}$ • Nielka P. van Erp ${ }^{6} \cdot$ Ron H. J. Mathijssen ${ }^{3} \cdot$ Jos H. Beijnen ${ }^{1,2,7}$ • \\ Alwin D. R. Huitema ${ }^{1,2,8}$
}

Received: 6 April 2019 / Accepted: 2 September 2019

(C) Springer Science+Business Media, LLC, part of Springer Nature 2019

\begin{abstract}
Purpose There is ongoing concern regarding increased toxicity from paclitaxel in elderly patients, particularly of severe neutropenia. Yet, data so far is controversial and this concern is not supported by a clinically relevant age-dependent difference in pharmacokinetics (PK) of paclitaxel. This study assessed whether age is associated with increased risk for paclitaxel-induced neutropenia.
\end{abstract}

Methods Paclitaxel plasma concentration-time data, pooled from multiple different studies, was combined with available respective neutrophil count data during the first treatment cycle. Paclitaxel pharmacokinetic-pharmacodynamic (PKPD) data was modeled using a non-linear mixed effects approach and a semiphysiological neutropenia model, where

Marie-Rose B. S. Crombag

m.crombag@nki.nl

Department of Pharmacy \& Pharmacology, Antoni van Leeuwenhoek Netherlands Cancer Institute, Plesmanlaan 121, 1066

CX Amsterdam, the Netherlands

2 Division of Pharmacology, Netherlands Cancer Institute, Amsterdam, the Netherlands

3 Department of Medical Oncology, Erasmus MC Cancer Institute, Rotterdam, the Netherlands

4 Department of Hospital Pharmacy, Erasmus MC, Rotterdam, the Netherlands

5 Department of Medical Oncology and Hematology, Cantonal Hospital, St Gallen, Switzerland

6 Department of Pharmacy and Radboud Institute of Health Sciences, Radboud University Medical Center, Nijmegen, the Netherlands

7 Utrecht Institute for Pharmaceutical Sciences (UIPS), Division of Pharmacoepidemiology and Clinical Pharmacology, Utrecht University, Utrecht, the Netherlands

8 Department of Clinical Pharmacy, University Medical Center Utrecht, Utrecht University, Utrecht, the Netherlands systemic paclitaxel exposure was linked to reduced proliferation of neutrophils. The impact of age was evaluated on relevant variables in the model, using a significance threshold of $p<0.005$.

Results Paclitaxel PK-PD data was evaluated from 300 patients, with a median age of 65 years (range 23-84 years), containing 116 patients $\geq 70$ years $(39 \%)$. First cycle neutrophil counts were adequately described by a threshold effect model of paclitaxel on the proliferation rate of neutrophils. Age as a continuous or dichotomous variable $(\geq 70$ versus $<70$ years) did not significantly impact sensitivity of the bone marrow to paclitaxel nor the average maturation time of neutrophils (both $p>0.005$ ), causing a decline in the respective interindividual variability of $<1 \%$.

Conclusion Results from this large retrospective patient cohort do not suggest elderly patients to be at an increased risk of developing paclitaxel-associated neutropenia during the first treatment cycle. Reflexive dose reductions of paclitaxel in elderly patients are unlikely to improve the risk of severe neutropenia and may be deleterious.

KEY WORDS age differences · elderly patients . neutropenia · paclitaxel · pharmacokinetics-pharmacodynamics

$\begin{array}{ll}\text { ABBREVIATIONS } \\ \text { ANC }_{\text {base }} & \text { Baseline absolute neutrophil count } \\ \text { ANC }_{t} & \text { Absolute neutrophil count at time } t \\ C_{\text {drug }} & \text { Systemic drug concentration } \\ \text { EC }_{50} & \text { Drug concentration at half the maximum } \\ & \text { inhibitory effect } \\ E_{\text {drug }} & \text { Drug effect } \\ E_{\max } & \text { Maximum inhibiting drug effect } \\ \text { Erasmus } & \text { Erasmus Medical Center Cancer Institute } \\ M C & \\ \text { FB } & \text { Feedback } \\ \text { IIV } & \text { Interindividual variability }\end{array}$




$\begin{array}{ll}k_{\text {prol }} & \text { Rate constant for the proliferation of neutrophils } \\ k_{\mathrm{tr}} & \text { Rate constant for the maturation of neutrophils } \\ \text { MTT } & \text { Mean transit time } \\ \mathrm{NKI} & \text { Netherlands Cancer Institute } \\ \text { PK-PD } & \text { Pharmacokinetic-pharmacodynamic } \\ \text { Radboud } & \text { Radboud University Medical Center } \\ \text { UMC } & \\ \text { SLOPE } & \text { Slope factor of linear drug effect } \\ \mathrm{T}_{\mathrm{c}>0.05 \mu \mathrm{M}} & \begin{array}{l}\text { Time-above-threshold concentration of } \\ \end{array} \\ & 0.05 \mu \text { mol/L }\end{array}$

\section{INTRODUCTION}

Chemotherapy remains the cornerstone of anticancer treatment and is frequently administered to the ever-growing elderly patient population. Dose finding of chemotherapy, including paclitaxel, has been based on identifying the maximum tolerated dose in a small cohort of patients to ultimately maximize its therapeutic effect in the intended patient population. The dose limiting toxicity of paclitaxel is neutropenia, which may lead to potentially fatal infections (1). Substantial heterogeneity in treatment-related toxicity has been reported, and limited available data suggested that elderly patients may be at an increased risk of paclitaxel-induced neutropenia (1-3), although data is controversial (1,2,4-7). Pivotal clinical trials included a relatively small fraction of elderly patients $(17 \%$ aged $\geq 65$ years $)$ and reported an overall higher incidence of severe neutropenia $\left(<0.5 * 10^{9} / \mathrm{L}\right)$ in elderly patients, which reached statistical significance in two Phase III trials (1). It has been postulated that an age-related difference may be driven by a difference in exposure, a depleted bone marrow reserve, a higher sensitivity to chemotherapy-related hematological toxicity, and/or a diminished capacity to regain homeostasis after bone marrow stress $(8,9)$. To pursue optimal treatment outcome, it is key to timely initiate treatment that is not too aggressive nor too conservative, as both may lead to a higher morbidity and mortality. A sound understanding of crucial factors that influence the pharmacokineticpharmacodynamic (PK-PD) relationship facilitates its optimal use by helping to identify those patient characteristics that may predispose patients to neutropenia, including older age. In previous studies, no clinically relevant impact of older age on the pharmacokinetics of paclitaxel has been established (10), whereas the time-above-threshold concentration of $0.05 \mu \mathrm{mol} / \mathrm{L}\left(\mathrm{T}_{\mathrm{c}}>0.05 \mu \mathrm{M}\right)$ has been shown to be related to paclitaxel-induced hematological toxicity $(11-13)$. Hypothetically, elderly patients may be at an increased risk of neutropenia due to increased bone marrow sensitivity rather than increased exposure to paclitaxel. We designed this study to elucidate the impact of older age on paclitaxelinduced neutropenia using a comprehensive pharmacokinetic-pharmacodynamic model that includes bone marrow function.

\section{METHODS}

\section{Data Collection}

Patients who were enrolled in multiple different previously reported studies were eligible for inclusion in the current database. Four of these studies were clinical trials (14-16) and one study concerned a prospective observational study in which patients aged 70 years or older were included (10). All patients were treated with intravenous paclitaxel at the Netherlands Cancer Institute (NKI; Amsterdam, the Netherlands), the Erasmus Medical Center Cancer Institute (Erasmus MC; Rotterdam, the Netherlands), and the Radboud University Medical Center (Radboud UMC; Nijmegen, the Netherlands). Paclitaxel plasma concentration-time data was available from all patients in this pooled cohort. A median number of $4 \mathrm{PK}$ samples per patient was drawn in this cohort, ranging from 1 to 20 samples per patient (10). A more detailed description of study and patient characteristics have been reported previously $(10,14-18)$. In the current analysis, patients were excluded from the database if no neutrophil counts or only baseline neutrophil counts were available, or if accurate paclitaxel dosing information, especially concerning dates of paclitaxel treatment, could not be obtained. Patient characteristics, underlying malignancy, and baseline laboratory values were available in the pooled dataset. The first administered paclitaxel dose and associated neutrophil counts per patient were extracted from electronic records. If the patient received combination chemotherapy, dosing information of concomitantly administered chemotherapy was added to the database. Neutrophil counts were determined at multiple time points during paclitaxel treatment, according to routine clinical care as defined by the clinical treatment protocol or study protocol. All paclitaxel containing regimes were administered according to standard procedures of the participating study centers, using standard premedication, fixed infusion times, and dose reduction guidelines. In this analysis, only neutrophil counts after the first treatment cycle were used. All studies were approved by the institutional ethics committees and were carried out in accordance with International Conference on Harmonisation (ICH) Guidelines for Good Clinical Practice (19).

\section{Population Pharmacokinetic Model}

Individual PK parameters were estimated using a previously developed 3-compartment model with saturable distribution and elimination (14). The individual subject PK parameters 
that were estimated in our previously published PK study were used as PK input in the current study (10). For most patients, paclitaxel PK sampling was performed during the first cycle, which coincides with the neutropenia data used for the modeling. However, for a subset of patients (within a prospective NKI study and a retrospective Erasmus MC study) sampling was performed in one of the later treatment cycles. In the current analysis, the PK parameters for this subsequent cycle were estimated and used as input for the neutropenia model for the first cycle.

\section{Semiphysiological Neutropenia Model}

A semiphysiological neutropenia model, including continuous neutrophil count data, was used which links the proliferating pool of neutrophils to the pool of circulating neutrophils using three transit compartments that mimick the time delay related to maturation of neutrophils (20). A first-order rate constant was estimated for the proliferation $\left(\mathrm{k}_{\mathrm{prol}}\right)$ and maturation $\left(\mathrm{k}_{\mathrm{tr}}\right)$ of neutrophils. This semiphysiological model, based on Friberg et al. (20), included a feedback loop, which represented the homeostatic response to the paclitaxel-induced reduction in circulating neutrophils. The feedback parameter describing the neutrophil count recovery after paclitaxel administration was described by the exponent of the feedback constant $(\boldsymbol{\gamma})$ of the baseline absolute neutrophil count $\left(\mathrm{ANC}_{\text {base }}\right)$ divided by the absolute neutrophil count at time $t\left(\mathrm{ANC}_{t}\right)$, according to $\left(\mathrm{ANC}_{\text {base }} / \mathrm{ANC}_{t}\right)^{\gamma}$. A chain of three transit compartments was used (21), thus including four transitions from proliferation to circulation of neutrophils. The mean transit time (MTT) represented the average maturation time from the proliferating stage to circulating neutrophils. The process of proliferation and maturation of neutrophils was described by the following equations:

$k_{t r}=\frac{4}{M T T}$

$k_{\text {prol }}=\left(k_{\text {tr }} * F B\right)-\left(k_{t r} * E_{\text {drug }}\right)$

where $k_{t r}$ represents the maturation rate of neutrophils, MTT represents the mean transit time, $k_{\text {prol }}$ represents the proliferation rate of neutrophils, $\mathrm{FB}$ represents feedback, being $\left(\mathrm{ANC}_{\text {base }} / \mathrm{ANC}_{t}\right)^{\gamma}$ and $E_{\text {drug }}$ representing the drug effect of paclitaxel. To evaluate the impact of paclitaxel exposure on neutrophil counts a linear drug effect (A) as well as a nonlinear drug effect (B) on $\mathrm{k}_{\text {prol }}$ was considered, as formulated by the following equations:

$E_{\text {drug } A}=S L O P E^{*} C_{\text {drug }}$

$E_{\text {drug } B}=\left(E_{\text {max }} * C_{\text {drug }}\right) /\left(E C_{50}+C_{\text {drug }}\right)$

with $E_{d r u g}$ representing the drug effect, $C_{d r u g}$ representing the systemic paclitaxel concentration, $E_{\max }$ representing the maximum inhibitory effect, which was fixed to 1 , and $E C_{50}$ being the paclitaxel concentration at half the maximum inhibitory effect.

Additionally, we evaluated a threshold model in which only a relevant effect of paclitaxel on $k_{\text {prol }}$ was assumed above a previously established threshold. Moreover, if the paclitaxel concentration was above this threshold, maximum effect was assumed (11-13,22-25). This was implemented as follows:

$E_{\text {drug } C}=\left(E_{\text {max }} * C_{\text {drug }}\right.$ Hill $) /\left(E C_{50}{ }^{\text {Hill }}+C_{\text {drug }}\right.$ Hill $)$

with Hill representing the gamma coefficient, which was fixed at 20 to create an extremely steep concentration-effect model with $\mathrm{EG}_{50}$ fixed on a predefined threshold total paclitaxel concentration of $0.05 \mu \mathrm{mol} / \mathrm{L}(11-13,22-25)$.

The drug effect from either one of the above-described eqs. $3-5$ was inserted into eq. 2 . It was assumed that $\mathrm{k}_{\text {prol }}$ equals $\mathrm{k}_{\mathrm{tr}}$ and that the transition rate was equal for transition through all three transit compartments, including transit to the central circulation compartment. Hereto, the following parameters were estimated: MTT, $\gamma$, and either SLOPE, $\mathrm{EC}_{50}$, or $\mathrm{E}_{\max }$ for eq. 3-5, respectively, as presented in Fig. 1.

As a relevant part of the patients received carboplatin infusions concomitantly, the effect of carboplatin on neutrophil counts was included. Since only carboplatin dosing information was available and PK data was lacking, typical PK-PD parameters of carboplatin were used from a previously published analysis by Joerger et al. (26). Hence, an additional linear drug effect using a slope factor for carboplatin was included, as presented in the above-described eq. 3. The following fixed parameter estimates were used; carboplatin clearance of $7.38 \mathrm{~L} / \mathrm{h}$, volume of the central compartment of $11.9 \mathrm{~L}$, volume of second peripheral compartment of $8.23 \mathrm{~L}$, intercompartmental clearance between central and second peripheral compartment of $5.43 \mathrm{~L} / \mathrm{h}$, and slope of $1.62 *$ $10^{-3} \mu \mathrm{mol} / \mathrm{L}(26)$. Because no other concomitant chemotherapy was used in the current study cohort, the additive effect of other cytostatic drugs was not considered.

In the neutropenia model, interindividual variability (IIV) was estimated on MTT and either on SLOPE, $\mathrm{EC}_{50}$, or $\mathrm{E}_{\max }$, respectively for assessment of the models described by eqs. 3 to 5 above. The residual error model was characterized using a proportional error model. Baseline neutrophil counts were estimated using the observed baseline observations as well as the residual variability (method B2 according to Dansirikul et al.) (27).

\section{Model Evaluation of Older Age}

The impact of older age was evaluated both as a continuous variable and as a dichotomous variable $\geq 70$ years versus $<70$ years). The effect of this covariate was evaluated on two $\mathrm{PD}$ parameters, namely either $\mathrm{SLOPE}, \mathrm{EC}_{50}$, or $\mathrm{E}_{\max }$, 


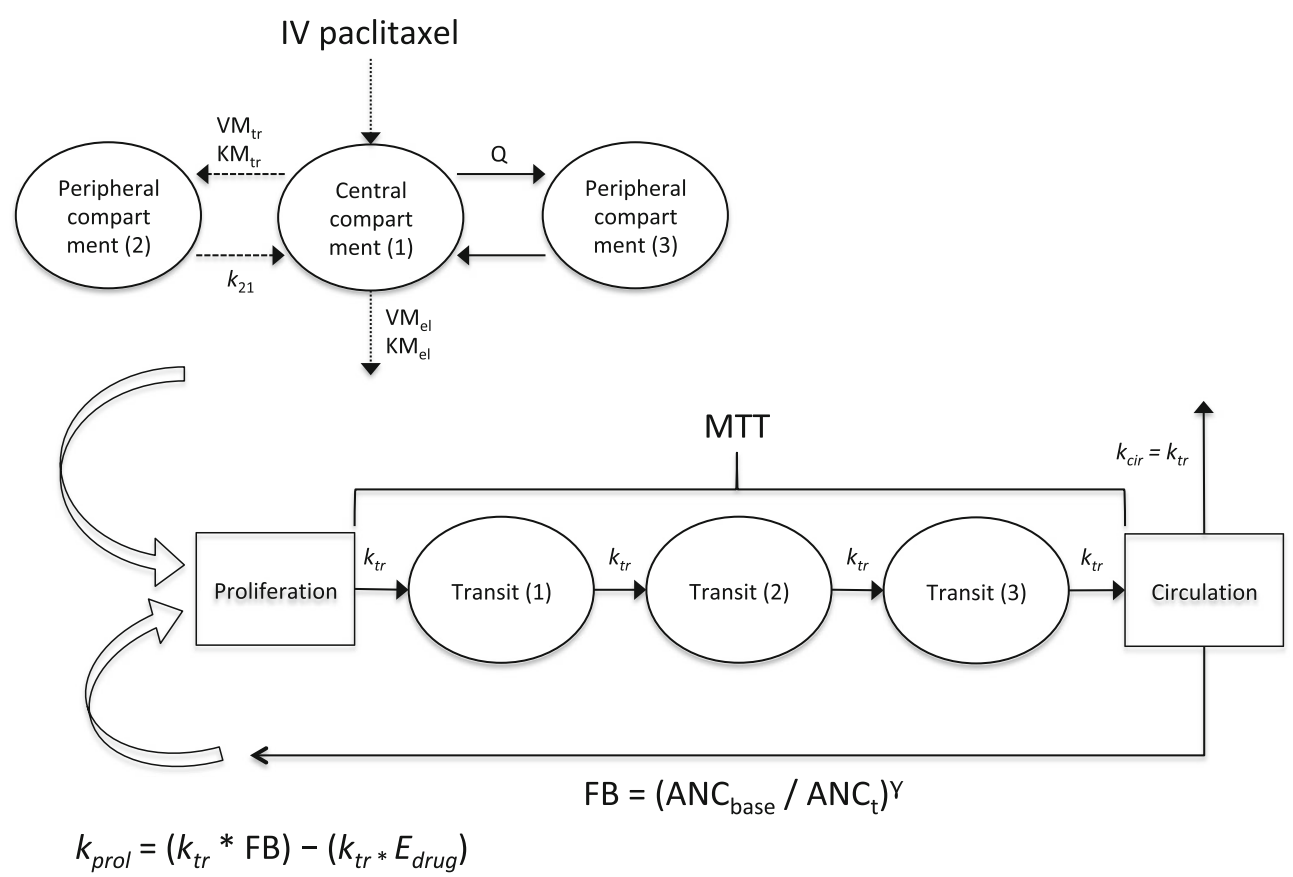

Fig. I Schematic presentation of the semiphysiological neutropenia model. Pharmacokinetic model: $K 2 \mathrm{I}=$ rate constant of the distribution from the first peripheral compartment to the central compartment, $\mathrm{KMel}=$ plasma concentration at half $\mathrm{VMel}$, KMtr $=$ plasma concentration at half $\mathrm{VM}$ tr, $\mathrm{Q}=$ intercompartmental clearance between the central and second peripheral compartment, VMel = maximal elimination rate, VMtr = maximal transport rate from the central to the first peripheral compartment. Pharmacodynamic model: ANCbase = baseline absolute neutrophil count, ANCt $=$ absolute neutrophil count at time $\mathrm{t}, \mathrm{Y}=$ exponent of the feedback constant, Edrug $=$ drug effect, $\mathrm{FB}=$ feedback, $\mathrm{kprol}=$ rate constant for the proliferation of neutrophils, $\mathrm{ktr}=$ rate constant for the maturation of neutrophils, MTT = mean transit time.

depending on the chosen final neutropenia model, and on MTT. For assessment of age as a continuous variable the following equation was applied:

$P D_{P A R}=\Theta_{\mid} *\left(\frac{A G E}{A G E_{\text {median }}}\right)^{\theta_{2}}$

where $\mathrm{PD}_{\mathrm{PAR}}$ represents the concerned PD parameter, e.g. $\mathrm{E}_{\max }, \Theta_{1}$ represents the typical population value for the PD parameter, AGE represents the continuous covariate age, centered to the median value, $\Theta_{2}$ represents the effect of age.

Assessment of the impact of age as a dichotomous variable was performed using the following equation:

$P D_{P A R}=\Theta_{1} * \Theta_{2}^{A G E D}$

where $\mathrm{PD}_{\mathrm{PAR}}$ again represents the concerned PD parameter, $\Theta_{1}$ represents the typical $\mathrm{PD}_{\mathrm{PAR}}$ value for patients aged $<70$ years, $\Theta_{2}$ represents the parameter estimate for the covariate effect, denoting the proportional increase or decrease for patients aged $\geq 70$ years, with AGED set 0 for younger and 1 for elderly patients. Furthermore, the impact of gender and performance status (PS) was also evaluated using eq. 7, with PS dichotomized into two groups, namely PS 0-1 and PS 2-3.

Model evaluation was performed by evaluating successful and plausible parameter estimation, model stability, goodness-of-fit (GOF) plots, Visual Predictive Check (VPG) evaluation with $n=1000$, changes in IIV, parameter precision, and drop in Objective Function Value (OFV) with a significance threshold of $p<0.005$ (dOFV $>7.9$ for 1 degree of freedom) for hierarchical models. Precision of parameter estimates was assessed using sampling importance resampling (SIR) (28). The relationship between $\mathrm{T}_{\mathrm{c}}>0.05 \mu \mathrm{M}$ and estimated paclitaxel-induced neutropenia was visualized with the final model parameters, including age both as a continuous variable and dichotomized into two age groups. Hereto, Bayesian estimates of $\mathrm{T}_{\mathrm{c}}>0.05 \mu \mathrm{M}$, obtained with the POSTHOC option in NONMEM, were used. Change from baseline neutrophil to nadir count was calculated also using the Bayesian estimates of nadir neutrophil count.

\section{Software}

Data handling, descriptive data analysis, and data plotting was performed using $\mathrm{R}$ (version 3.0.1). For descriptive analysis, the Wilcoxon rank sum test and Fisher's exact test were used, with the significance threshold set at $p<0.05$. Non-linear mixed effects modeling was executed by NONMEM ${ }^{\circledR}$ (version 7.3.0, ICON Development Solutions, Ellicott City, MD, USA) and Perl-speaks-NONMEM (version 4.4.8). The first order conditional estimation with interaction was used as estimation method, with Pirana (version 2.9.2) used as a model interface. 


\section{RESULTS}

Paclitaxel plasma concentration-neutrophil count data of 300 patients was included, with a total of 825 neutrophil count measurements, of which 300 were baseline measurements, as presented in Table I. Overall, a median number of 2 neutrophil count measurements per patient was available, ranging from one to eight neutrophil count measurements per patient, with no measurements below the limit of quantitation. Median age of the total cohort of patients was 65 years, ranging from 23 to 84 years, and 116 patients were aged $\geq 70$ years (39\%). Our cohort comprised 221 patients who were enrolled in previous clinical trials, and 79 patients aged 70 years or older who were included in a previously reported prospective observational study in daily clinical practice (10). The majority of patients received paclitaxel in combination with carboplatin $(88 \%)$. Most frequently recorded malignancies were upper gastro-intestinal and gynaecological cancers. Relatively more elderly patients were male and received paclitaxel in a weekly monotherapy regimen compared to their younger counterparts receiving 3-weekly treatment, as depicted in Table I. Remarkably, elderly patients had a significantly higher baseline neutrophil count compared to their younger counterparts ( $p<0.001$, median 5.8 versus $4.4 * 10^{9} / \mathrm{L}$, respectively), as depicted in Table I. Baseline neutrophil counts were available for all patients included in the current analysis.

\section{Paclitaxel-Induced Neutropenia}

During the first treatment cycle, significantly fewer neutrophil counts were measured in elderly compared to younger patients $(\mathrm{p}<0.001)$, which was especially evident in the 3weekly cohort. Conforming to local treatment protocols nadir values were generally missing in the prospectively included cohort of elderly patients treated with 3-weekly paclitaxel in routine clinical practice.

The paclitaxel-neutrophil relationship was evaluated using the aforementioned slope, non-linear $\mathrm{E}_{\max }$ type and the threshold models. All three models were able to fit neutrophil count data and showed plausible parameter estimates. A large IIV of $>50 \%$ was observed in each model for SLOPE $(80 \%)$, $\mathrm{EC}_{50}(220 \%)$, or $\mathrm{E}_{\max }(67 \%)$. While all three models estimated a total number of six parameters, the threshold model had the lowest $\mathrm{OFV}$, with a drop of 35 and 2 points as compared to the SLOPE and $\mathrm{EG}_{50}$ model, respectively. Model stability, goodness-of-fit plots, and parameter precision were superior for the threshold model. Therefore, the threshold model with a predefined threshold concentration was considered more appropriate to describe paclitaxel plasma concentrationneutrophil-time data. Figure 2 shows the observed neutrophil counts versus population predicted and individual predicted neutrophil counts by this threshold model. In Fig. 3 the VPG evaluation with $n=1000$ is presented. Parameter uncertainty distribution was evaluated using SIR, which showed that estimates had sufficient precision, with the 95\% confidence intervals (CI) depicted in Table II.

In the selected threshold model, the impact of age on paclitaxel-induced neutropenia was evaluated. Age treated as a continuous variable did not significantly influence $\mathrm{E}_{\max }$ or MTT (both $p>0.005$ ). The relative standard error (RSE) of the effect of age on $\mathrm{E}_{\max }$ was $68 \%$, whereas the RSE of the effect of age on MTT was $53 \%$. The reduction in IIV of either $\mathrm{E}_{\max }$ or MTT was $<1 \%$, with a shrinkage of $30 \%$ and $32 \%$, respectively. Evaluation of age handled as dichotomized variable showed similar results on $\mathrm{E}_{\max }$ or MTT (both $\mathrm{p}>0.005$ ). In Fig. $4, \mathrm{~T}_{\mathrm{c}}>0.05 \mu \mathrm{M}$ was shown against the estimated relative neutropenia during the first treatment cycle, with age depicted continuously and dichotomized into two age groups $\geq 70$ versus $<70$ years). This graph clearly showed the relation between $\mathrm{T}_{\mathrm{c}}>0.05 \mu \mathrm{M}$ and paclitaxel-induced neutropenia. Introduction of the covariates gender and PS had no significant effect on neutropenia.

\section{DISCUSSION}

In this comprehensive study cohort, including a substantial fraction of elderly patients, older age was not significantly related to the risk of developing paclitaxel-induced neutropenia. Gender and PS also did not significantly influence neutropenia in our study analysis.

Given the small therapeutic window of paclitaxel, extensive interpatient variability in PK-PD, and hazardous toxicity profile, there remains concern on its safety profile in elderly patients. As acknowledged by the regulatory authorities, severe neutropenia was more frequently observed in the elderly patients, but this difference did not reach significance in the majority of reported pivotal trials (1). Similarly, conflicting results are published regarding patients treated in daily clinical practice. One study reported a significantly higher risk of severe neutropenia in elderly patients (2), although the authors also stated that this was not accompanied by a significant difference in clinical consequences as hospitalization, fever, or need for intravenous antibiotics. Other studies observed no significant impact of older age on paclitaxel-induced toxicity (4-7). One of these studies was a relatively large study $(n=251)$ and showed no impact of older age, although the patient cohorts were younger than the current study (median 58 to 60 years) (7). The other studies included a rather small number of patients. In one of these studies the younger patient control group was lacking (5), whereas in another study elderly patients received lower paclitaxel doses than their younger counterparts (6). Our study cohort included an extensive and heterogeneous group of both younger and elderly patients. Elderly patients had a PS ranging from 0 to 3 and they were enrolled in clinical trials or treated as part of daily clinical 
Table I Baseline Patients' Characteristics

\begin{tabular}{|c|c|c|c|c|}
\hline Parameter & Total cohort & Aged $<70$ yrs & Aged $\geq 70$ yrs & $p$ value \\
\hline Number of patients, $n$ (\%) & $300(100)$ & $184(100)$ & $116(100)$ & \\
\hline Weekly regimen & $172(57)$ & $80(43)$ & $92(79)$ & \\
\hline 3-weekly regimen & $128(43)$ & $104(57)$ & $24(21)$ & \\
\hline Age $(y)$, median [range] & $65[23-84]$ & 58 [23-69] & $73[70-84]$ & \\
\hline \multicolumn{5}{|l|}{ Treatment regimen, (n) } \\
\hline Monotherapy & 34 & 2 & 32 & \\
\hline With carboplatin & 263 & 179 & 84 & $<0.001$ \\
\hline Unknown & 3 & 3 & 0 & \\
\hline \multicolumn{5}{|l|}{ Paclitaxel dose $\left(\mathrm{mg} / \mathrm{m}^{2}\right)$, median [range] } \\
\hline Weekly regimen & $51[38-103]$ & $51[47-103]$ & $51[38-101]$ & 0.41 \\
\hline 3-weekly combination & 173 [130-203] & $173[16 \mid-203]$ & 173 [130-179] & 0.56 \\
\hline No. of post baseline observations ( $n$ ) & 525 & 385 & 140 & \\
\hline Per patient, median [range] & | [1-7] & $2[1-7]$ & | [1-4] & $<0.001$ \\
\hline Weekly regimen & $\mid[1-3]$ & $\mid[1-2]$ & $\mid[\mid-3]$ & 0.20 \\
\hline 3-weekly regimen & $3[1-7]$ & $3[1-7]$ & | $[1-4]$ & $<0.001$ \\
\hline \multicolumn{5}{|c|}{ Sampling time of post baseline observations (days), median [range] } \\
\hline All & $10[2-45]$ & $\mid 3[4-4 \mid]$ & $7[2-45]$ & $<0.001$ \\
\hline Weekly regimen & $7[2-20]$ & $7[5-14]$ & $7[2-20]$ & 0.50 \\
\hline 3-weekly regimen & $14[4-45]$ & $\mid 4[4-4 \mid]$ & $20[5-45]$ & 0.002 \\
\hline \multicolumn{5}{|l|}{ Female } \\
\hline$n,(\%)$ & $186(62)$ & $124(67)$ & $62(53)$ & 0.02 \\
\hline \multicolumn{5}{|l|}{ Indication, $n$ (\%)* } \\
\hline Upper Gl & $134(45)$ & $76(4 I)$ & $58(50)$ & \\
\hline Gynaecological & $|2|(40)$ & $106(58)$ & $15(13)$ & \\
\hline Breast & $31(10)$ & $2(1)$ & $29(25)$ & \\
\hline Urological & $10(3)$ & 0 & $10(9)$ & \\
\hline Other & $3(1)$ & 0 & $3(3)$ & \\
\hline Unknown & I (0.3) & 0 & $\mathrm{I}(\mathrm{I})$ & \\
\hline \multicolumn{5}{|l|}{ Hospital } \\
\hline NKI & 179 & 97 & 82 & \\
\hline Erasmus MC & 117 & 87 & 30 & \\
\hline Radboud UMC & 4 & 0 & 4 & \\
\hline \multicolumn{5}{|l|}{ Baseline laboratory values } \\
\hline \multicolumn{5}{|l|}{ Neutrophil counts $\left(* 10^{9} / L\right)$, median $[I Q R]$} \\
\hline All & $4.7[3.6-6.6]$ & $4.4[3.5-5.9]$ & $5.8[4.2-7.4]$ & $<0.001$ \\
\hline Weekly regimen & $4.8[3.9-6.9]$ & $4.4[3.8-6.1]$ & $5.5[4.1-7.2]$ & 0.02 \\
\hline 3-weekly regimen & $4.6[3.4-6.1]$ & $4.2[3.2-5.8]$ & $6.5[5.5-7.7]$ & $<0.001$ \\
\hline $\operatorname{BSA}\left(\mathrm{m}^{2}\right)$, median $[\mathrm{IQR}]$ & $1.8[1.6-2.0]$ & $1.8[1.6-1.9]$ & $1.8[1.7-2.0]$ & 0.01 \\
\hline \multicolumn{5}{|l|}{ Performance Status, $n$ (\%)* } \\
\hline $0-1$ & $264(88)$ & |6| (88) & $103(89)$ & 0.38 \\
\hline $2-3$ & $13(4)$ & $10(5)$ & $3(3)$ & \\
\hline Unknown & $23(8)$ & $13(7)$ & $10(9)$ & \\
\hline
\end{tabular}

* percentages do not add up to $100 \%$ due to rounding

BSA = body surface area, Gynaecological = ovarian, endometrium, mullarian, cervix, and vaginal cancer, $h=$ hours, IQR $=$ interquartile range 25th - 75th percentile, $\mathrm{m} 2$ = squared meter, $\mathrm{mg}=$ milligrams, $\mathrm{n}=$ number of patients, Upper $\mathrm{Gl}=$ esophageal and cardia cancer, Urological = testis, bladder, prostate, and kidney cancer, $y=$ years 

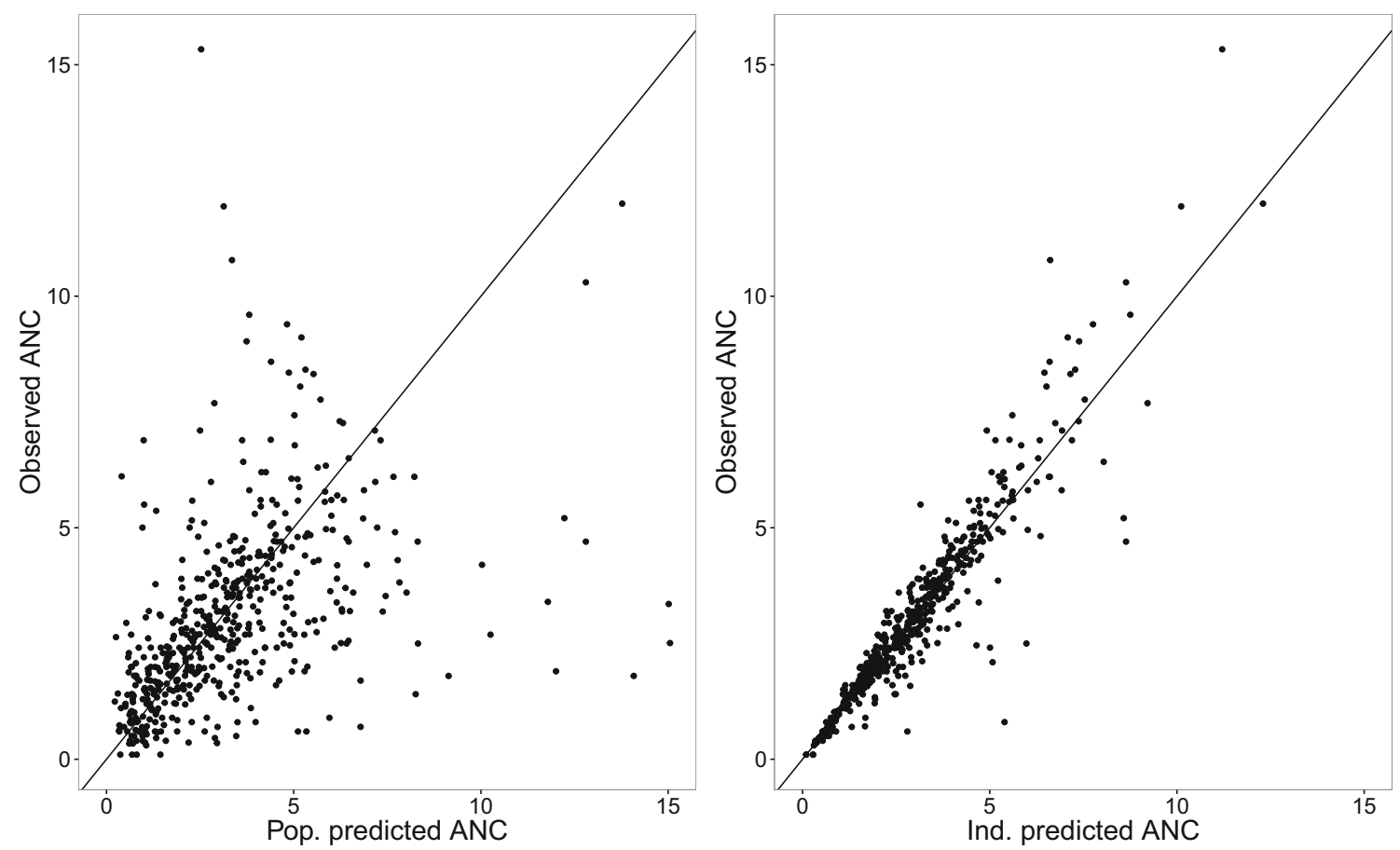

Fig. 2 Observed absolute neutrophil counts (ANC) versus population predicted and individual predicted ANC.

practice. Whilst considering the statistically significant agerelated difference in PK, which was not considered clinically relevant (i.e. a 5\% decrease in maximal elimination capacity for a 10-year increment of age for a typical patient) $(10,14)$, this comprehensive study showed no significant impact of older age on the risk of incurring paclitaxel-induced neutropenia.

$\mathrm{T}_{\mathrm{c}}>0.05 \mu \mathrm{M}$ has been shown to be related to paclitaxel treatment efficacy, neuropathy, and hematological toxicity (11-13,15,16,22-25). Previous studies described a linear drug effect on neutropenia $(14,21)$. In this study, paclitaxel plasma concentration-neutrophil count data was adequately described by a threshold model using this predefined threshold concentration of $0.05 \mu \mathrm{mol} / \mathrm{L}$. Our data showed no significant impact of older age on either $\mathrm{E}_{\max }$ or MTT, suggesting that elderly patients were not more sensitive to paclitaxelinduced neutropenia nor that recovery from hematological stress was delayed in elderly patients. This study only focused on neutrophil count data because we had no sound information on the occurrence of neutropenic fever. Although the latter may be of special interest for treating physicians, paclitaxel treatment adjustments are based on neutrophil count measurements in order to prevent neutropenic complications. Previous studies have consistently shown that first-cycle neutropenia is a good predictor of neutropenic complications in later treatment cycles (14,30-32). In absence of an effect of older age on first-cycle neutropenia, it is therefore not expected that there is an age-related difference in clinical consequences during later treatment cycles. However, some patients may not have a significant reduction of neutrophil counts during the first cycle, but may develop gradual reduction in neutrophil counts after multiple treatment cycles. It cannot be ruled out that a negligible age-related difference during the first treatment cycle may be pursued by a larger difference during the last treatment cycles. Nevertheless, inclusion of subsequent cycles may also introduce bias, as it may appear that neutropenia becomes less severe over multiple cycles due to the drop out of patients who develop severe neutropenia in earlier treatment cycles. Furthermore, the risk of developing peripheral neuropathy was not within the scope of our study. Although this adverse event was not defined as the major dose-limiting toxicity, it can be a severe and debilitating, especially for elderly patients (1). Neurotoxicity appeared to be cumulative over time, but unfortunately this information could not be retrieved reliably from all records of patients included in our cohort.

To provide a forward presentation and interpretation of the influence of older age, age was assessed as a continuous and as a dichotomized variable using a cut-off value of 70 years. This cut-off value was used because multiple previous studies showed a drastic increase in the incidence and severity of chemotherapy-induced hematological toxicity after the age of 70 (8). The current study aimed to include all available paclitaxel PK-PD data from multiple previous studies. As a consequence some differences between elderly and younger patients could not be averted. Interestingly, elderly patients in the study cohort had a significantly higher median baseline neutrophil count compared their younger counterparts. This may be due to the inclusion criteria of historic clinical trials in which these younger patients were originally included. Per 
Fig. 3 Visual Predictive Check. Visual Predictive Check with $n=$ I 000 of post baseline neutrophil counts, with the dots representing the observed neutrophil counts, the solid line representing observed median counts, and dashed lines representing the observed 5 th and 95th percentiles. The blue areas indicate the $95 \%$ confidence intervals.

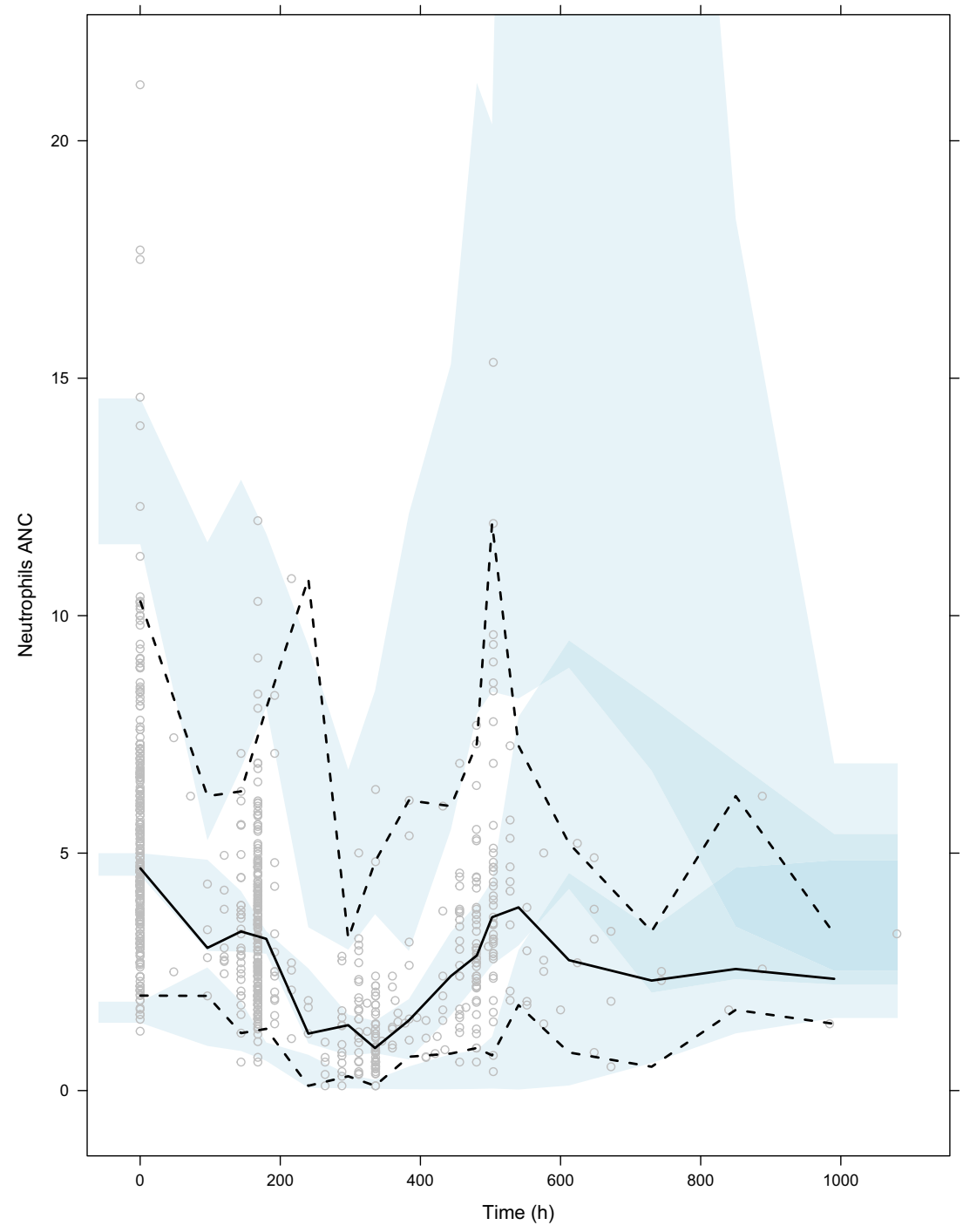

protocol, these trial participants generally have received more myelosuppressive chemotherapy or more lines of systemic treatment prior to starting paclitaxel. Besides, cigarette-

Table II PD parameter estimates of the final neutropenia model

\begin{tabular}{lcl}
\hline Parameter & Estimate & $\begin{array}{l}\text { 95\% Confidence interval } \\
\text { (RSE) [shrinkage] }\end{array}$ \\
\hline MTT (h) & 158 & $\mid 52-166(2)$ \\
$E_{\max }(\mu \mathrm{mol} / \mathrm{L})$ & 2.85 & $2.57-3.3 \mid(6)$ \\
Gamma & 0.24 & $0.22-0.25(4)$ \\
Interindividual variability & & $\mid 6-20(13)(32)$ \\
MTT (\%) & 18 & $58-77(14)(30)$ \\
$E_{\max }(\%)$ & 67 & \\
Residual error & & $44-46(3)(24)$ \\
$\sigma_{\text {prop }}(\%)$ & 45 &
\end{tabular}

$\mathrm{E}_{\max }=$ maximum inhibiting drug effect, $\mathrm{h}=$ hour, $\mathrm{MTT}=$ mean transit time, $\mathrm{RSE}=$ relative standard error, $\sigma_{\text {prop }}=$ proportional residual error smoking patients appeared to have a higher baseline neutrophil count compared to nonsmokers (29). Contrary to our findings, baseline neutrophil counts were higher in younger patients in this previous study and smokers were significantly younger than nonsmokers. However, adequate information about prior lines of chemotherapy and smoking status were not available in the current cohort. Hence, their contribution to the observed difference in baseline neutrophil counts could not be assessed in this study. This is a limitation of the current study, as a potential bias of prior exposure to myelosuppressive chemotherapy cannot be ruled out. The baseline neutrophil count of all patients was available and residual variability was taken into account in this model. However, the potential effect of covariates on this baseline value was not evaluated (27). Since elderly patients in this cohort had a higher baseline value, it is not likely that the conclusions on the probability of neutropenia would be altered. Nevertheless, it should be noted that median neutrophil counts at baseline were well within the normal range in both age groups. In line with the study 

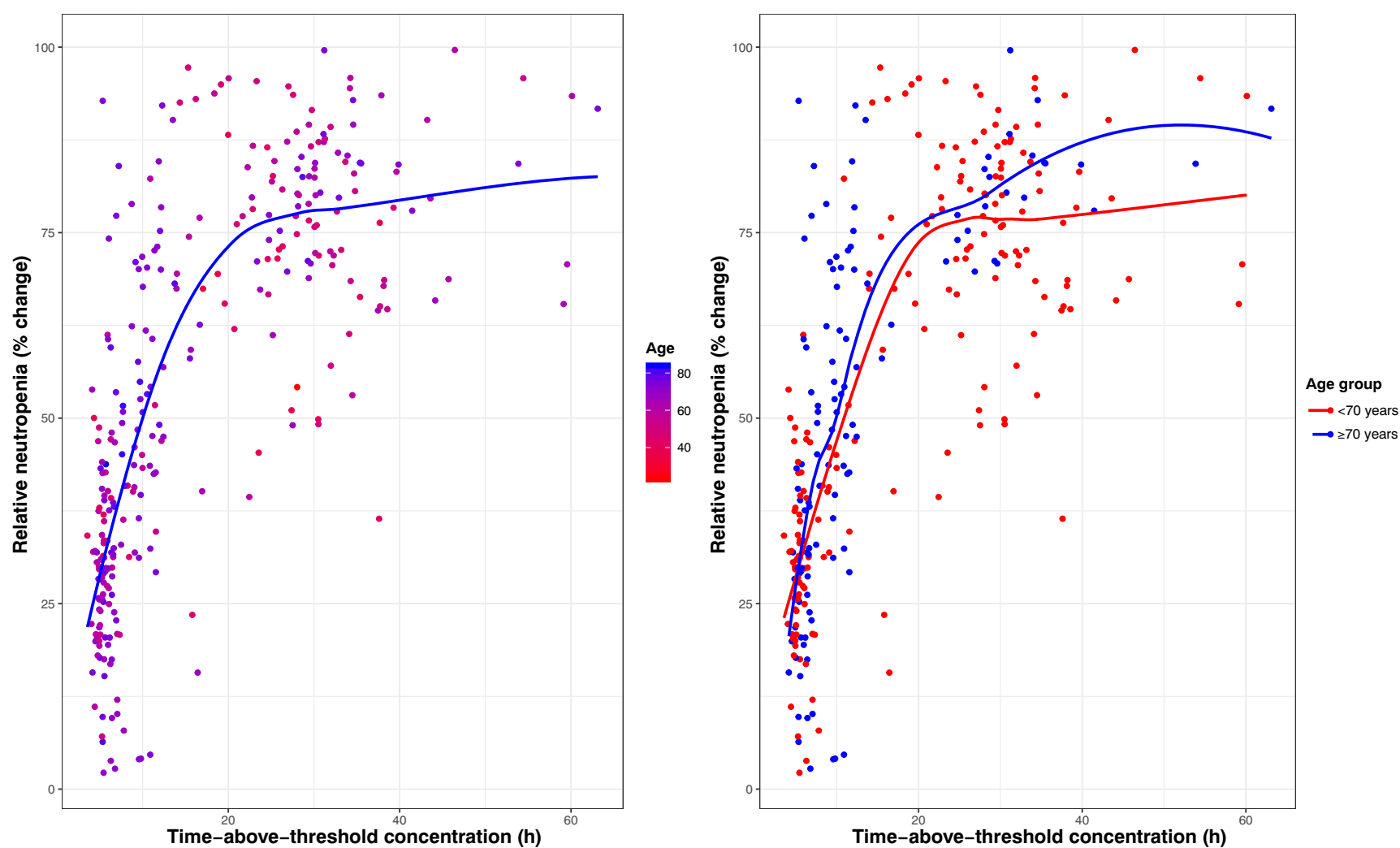

Fig. 4 Time-above-threshold paclitaxel concentration of $0.05 \mu \mathrm{mol} / \mathrm{L}$ versus estimated neutrophil count decline (\% change) depicted by age. With time-abovethreshold concentration of $0.05 \mu \mathrm{mol} / \mathrm{L}$ depicted in hours and estimated neutrophil count decline calculated as the percentage change in neutrophil count from baseline to nadir per patient. Age was depicted in years, displayed both as a continuous and as a dichotomous variable ( $\geq 70$ versus $<70$ years).

design of retrospectively included clinical trials that included many younger patients, the younger patient group contained more female patients receiving 3-weekly paclitaxel administrations. Per protocol, nadir counts were closely monitored in these clinical trial cohorts, whereas neutrophil counts of patients treated in routine clinical practice were generally only monitored at each hospital visit. For the weekly regimen, the planned hospital visit nearly coincided with the nadir value. Yet, in the 3-weekly cohort, data from patients treated in daily clinical practice was relatively sparse and nadir counts were not routinely measured in clinical practice. In the semiphysiological neutropenia model differences in e.g. paclitaxel dose and timing of neutrophil count data were considered. Model evaluation did not reveal a significant impact of older age on the risk of developing neutropenia.

For a part of the study cohort PK sampling was not performed during the first treatment cycle but was performed during one of the later treatment cycles. In this study, the PK parameters for this subsequent cycle were estimated and used as input for the neutropenia model for the first treatment cycle. It should be noted, however, that this approach did not consider interoccasion variability, which was $16 \%$ for the elimination capacity of paclitaxel in the previous PK study (10). This patient cohort did not include other paclitaxel- based formulations, e.g. albumin-bound paclitaxel. Therefore, the estimates of this PK-PD model may not predict the probability of neutropenia of other paclitaxel formulations. Paclitaxel is rarely administered in monotherapy and this study included different paclitaxel treatment regimens administered either in monotherapy or, for the majority of included patients, concomitantly with carboplatin treatment. Previous studies reported no influence of carboplatin on the PK of paclitaxel $(33,34)$. Carboplatin may, however, contribute to the development of neutropenia, although the effect of carboplatin on neutrophil counts appeared minimal $(1.62 *$ $\left.10^{-3} \mu \mathrm{mol} / \mathrm{L}\right)(26)$. Because of mechanistic plausibility, and to preclude bias, we added a drug effect of carboplatin to the neutropenia model based on a previously developed linear drug effect model. Because no PK data of carboplatin was available, the PK-PD parameters of carboplatin were fixed (26). Renal clearance was not considered in this previous study, but given the small effect of carboplatin on neutropenia, it is not expected that this may thwart our findings. Other covariates besides age may influence the risk of developing neutropenia, such as gender and PS. In a previous study, females were more likely to develop paclitaxel-induced neutropenia (7). However, in the current study gender did not significantly affect neutropenia, which is in line with the drug 
label (1). It should also be kept in mind that gender significantly influenced paclitaxel PK and that this effect was already incorporated in the current PK-PD analysis (i.e. males had a $12 \%$ higher maximal elimination capacity) (10). One may argue that not older age but PS or a combination of both may have predictive value for chemotherapy-induced toxicity. Although PS is commonly used to predict chemotherapyinduced toxicity in cancer patients, this is not validated in elderly patients. Hurria et al. determined no association between PS and treatment toxicity in elderly patients (35). To preclude bias, we assessed the influence of PS on neutropenia. Our analysis supported the findings by Hurria $e t$ al. that PS did not significantly affect neutropenia. Validated tools, including various questionnaires, are available to predict chemotherapy-induced toxicity in elderly patients (35), but these are generally not implemented in clinical practice and were not available for patients included in the current dataset.

In conclusion, the paclitaxel plasma concentrationneutropenia relationship was adequately described by a threshold model, using a predefined threshold concentration of $0.05 \mu \mathrm{mol} / \mathrm{L}$. Results from this comprehensive retrospective patient cohort do not suggest elderly patients to be at an increased risk of paclitaxel-associated neutropenia. A priori dose reductions of paclitaxel in elderly patients are unlikely to improve the risk of severe neutropenia and may impair treatment outcome.

\section{ACKNOWLEDGMENTS AND DISCLOSURES}

The authors wish to express their gratitude to all study participants and health care employees involved in patient inclusion, with special thanks to Ellen van der Pan and Anoek van Straten. The authors thank the Research High Performance Computing facility of the NKI for support in the use of computational resources. Jos H. Beijnen is a (parttime) employee and shareholder of Modra Pharmaceuticals, and (partly) holds a patent on oral taxane pharmaceutical formulations. The other authors declare no conflicts of interest in connection with this manuscript. All procedures performed in studies involving human participants were in accordance with the ethical standards of the institutional research committees and was carried out in accordance with ICH Guidelines for Good Clinical Practice. Written informed consent was obtained from all individual participants.

\section{REFERENCES}

1. Food and Drug Administration. TAXOL (paclitaxel), NDA 020262. 2011.

2. Lichtman SM, Hollis D, Miller A A, Rosner GL, Rhoades C A, Lester EP, et al. Prospective evaluation of the relationship of patient age and paclitaxel clinical pharmacology: Cancer and leukemia group B (CALGB 9762). J Clin Oncol 2006;24:1846-1851.
3. Lichtman SM, Hurria A, Girrincione GT, Seidman AD, Winer E, Hudis C, et al. Paclitaxel efficacy and toxicity in older women with metastatic breast cancer: combined analysis of calgb 9342 and 9840. Ann Oncol. 2012;23:632-8.

4. Nakamura Y, Sekine I, Furuse K, Saijo N. Retrospective comparison of toxicity and efficacy in phase II trials of 3-h infusions of paclitaxel for patients 70 years of age or older and patients under 70 years of age. Cancer Chemother Pharmacol. 2000;46:114-8.

5. Fidias P, Supko JG, Martins R, Boral A, Carey R, Grossbard M, et al. Measurement and impact of co-morbidity in elderly patients with advanced non-small cell lung cancer treated with chemotherapy. A phase II study of weekly paclitaxel. Clin Cancer Res. 2001;7: 3942-9.

6. Smorenburg CH, Ten Tije AJ, VerweijJ, Bontenbal M, Mross K, Van Zomeren DM, et al. Altered clearance of unbound paclitaxel in elderly patients with metastatic breast cancer. Eur J Cancer. 2003;39:196-202.

7. Nieuweboer AJ, Smid M, de Graan A-JM, Elbouazzaoui S, de Bruijn P, Martens JW, et al. Predicting paclitaxel-induced neutropenia using the DMET platform. Pharmacogenomics. 2015;16: $1231-41$.

8. Sehl M, Sawhney R, Naeim A. Physiologic aspects of aging: impact on cancer management and decision making, part II. Cancer J. 2005; 11:461-73.

9. Aapro M, Skacel T, von Minckwitz G, Lawrinson S, Schwenkglenks M, Lyman GH, et al. Pegfilgrastim primary prophylaxis vs. current practice neutropenia management in elderly breast cancer patients receiving chemotherapy. Crit Rev Oncol Hematol. 2009;74:203-10.

10. Crombag M-RB, de Vries SA, Koolen SL, Wijngaard S, Joerger $\mathrm{M}$, Schellens JH, et al. Impact of older age on the exposure of paclitaxel: a population pharmacokinetic study. Pharm Res. 2019;36.

11. Gianni BL, Kearns GM, Giani A, Capri G, Vigan L, Locatelli A, et al. Nonlinear pharmacokinetics and metabolism of paclitaxel and its pharmacokinetic/Pharmacodynamic relationships in humans. J Clin Oncol. 1995;13:180-90.

12. Ohtsu T, Sasaki Y, Tamura T, Nishiwaki Y, Saijo N. Clinical pharmacokinetics and pharmacodynamics of paclitaxel: A 3-hour infusion versus a 24-hour infusion. Clin Cancer Res. 1995;1:599606.

13. Xin D, Zhou L, Li C, Zhang S, Huang H, Qiu G, et al. TC > 0.05 as a pharmacokinetic parameter of paclitaxel for therapeutic efficacy and toxicity in Cancer patients. Recent Pat Anticancer Drug Discov. 2018;13:341-7.

14. Joerger M, Kraff S, Huitema ADR, Feiss G, Moritz B, Schellens JHM, et al. Evaluation of a pharmacology-driven dosing algorithm of 3-weekly paclitaxel using therapeutic drug monitoring: A pharmacokinetic-pharmacodynamic simulation study. Clin Pharmacokinet. 2012;51:607-17.

15. De Graan AJM, Elens L, Smid M, Martens JW, Sparreboom A, Nieuweboer AJM, et al. A pharmacogenetic predictive model for paclitaxel clearance based on the DMET platform. Clin Cancer Res. 2013;19:5210-7.

16. De Graan AJM, Elens L, Sprowl JA, Sparreboom A, Friberg LE, Van Der Holt B, et al. CYP3A4*22 genotype and systemic exposure affect paclitaxel-induced neurotoxicity. Clin Cancer Res. 2013;19:3316-24.

17. Joerger M, Huitema ADR, van den Bongard DHJG, Schellens JHM, Beijnen JH. Quantitative effect of gender, age, liver function, and body size on the population pharmacokinetics of paclitaxel in patients with solid tumors. Clin Cancer Res. 2006;12:2150-7.

18. Kraff S, Nieuweboer AJM, Mathijssen RHJ, Baty F, De Graan AJ, Van Schaik RHN, et al. Pharmacokinetically based dosing of weekly paclitaxel to reduce drug-related neurotoxicity based on a single sample strategy. Cancer Chemother Pharmacol. 2015;75:975-83. 
19. The International Conference on Harmonisation of Technical Requirements for Registration of Pharmaceuticals for Human Use. (ICH) 2018. www.ich.org.

20. Friberg LE, Henningsson A, Maas H, Nguyen L, Karlsson MO. Model of chemotherapy-induced myelosuppression with parameter consistency across drugs. J Clin Oncol. 2002;20:4713-21.

21. Henrich A, Joerger M, Kraff S, Jaehde U, Huisinga W, Kloft C, et al. Semimechanistic bone marrow exhaustion pharmacokinetic/ Pharmacodynamic model for chemotherapy-induced cumulative neutropenia s. J Pharmacol Exp Ther J Pharmacol Exp Ther. 2017;362:347-58.

22. Mielke S, Sparreboom A, Steinberg SM, Gelderblom H, Unger C, Behringer D, et al. Association of Paclitaxel Pharmacokinetics with the development of peripheral neuropathy in patients with advanced Cancer. Clin Cancer Res. 2005;11:4843-50.

23. Mielke S, Mross K, Gerds T A, Schmidt A, Wäsch R, Berger DP, et al. Comparative neurotoxicity of weekly non-break paclitaxel infusions over 1 versus 3 h. Anti-Cancer Drugs 2003;14:785-792.

24. Joerger M, Von Pawel J, Kraff S, Fischer JR, Eberhardt W, Gauler TC. Open-label, randomised study of individualized, pharmacokinetically (PK)-guided dosing of paclitaxel combined with carboplatin or cisplatin in patients with advanced non-small cell lung cancer (NSCLC). Ann Oncol. 2016;27:1895-902.

25. Mielke S, Sparreboom A, Behringer D, Mross K. Paclitaxel pharmacokinetics and response to chemotherapy in patients with advanced cancer treated with a weekly regimen. Anticancer Res. 2005;25:4423-7.

26. Joerger M, Huitema ADR, Richel DJ, Dittrich C, Pavlidis N, Briasoulis E, et al. Population pharmacokinetics and pharmacodynamics of paclitaxel and carboplatin in ovarian cancer patients: A study by the European organization for research and treatment of cancer-pharmacology and molecular mechanisms group and new drug development group. Clin Cancer Res. 2007;13:6410-8.

27. Dansirikul C, Silber HE, Karlsson MO. Approaches to handling pharmacodynamic baseline responses. J Pharmacokinet Pharmacodyn. 2008;35:269-83. https://doi.org/10.1007/ s10928-008-9088-2.

28. Dosne AG, Bergstrand M, Harling K, Karlsson MO. Improving the estimation of parameter uncertainty distributions in nonlinear mixed effects models using sampling importance resampling. J Pharmacokinet Pharmacodyn. 2016;43:583-96.

29. de Graan A-JM, Loos WJ, Friberg LE, Baker SD, van der Bol JM, van Doorn L, et al. Influence of smoking on the pharmacokinetics and toxicity profiles of Taxane therapy. Clin Cancer Res. 2012;18: 4425-32.

30. Fontanella C, Bolzonello S, Lederer B, Aprile G. Management of breast cancer patients with chemotherapy-induced neutropenia or febrile neutropenia. Breast Care. 2014;9:239-45.

31. Pettengell R, Schwenkglenks M, Leonard R, Bosly A, Paridaens R, Constenla M, et al. Neutropenia occurrence and predictors of reduced chemotherapy delivery: results from the INC-EU prospective observational European neutropenia study. Support Care Cancer. 2008;16:1299-309.

32. Rivera E, Haim Erder M, Fridman M, Frye D, Hortobagyi GN. First-cycle absolute neutrophil count can be used to improve chemotherapy-dose delivery and reduce the risk of febrile neutropenia in patients receiving adjuvant therapy: a validation study. Breast Cancer Res. 2003;5:114-20. https://doi.org/10.1186/ bcr618.

33. Belani BCP, Kearns CM, Zuhowski EG, Erkmen K, Hiponia D, Zacharski D, et al. Phase I Trial , Including Pharmacokinetic and Pharmacodynamic Correlations, of Combination Paclitaxel and Carboplatin in Patients With Metastatic Non-Small-Cell Lung Cancer. J Clin Oncol 1999;17:676-684

34. Huizing MT, van Warmerdam LJ, Rosing H, Schaefers MC, Lai A, Helmerhorst TJ, et al. Phase I and pharmacologic study of the combination paclitaxel and carboplatin as first-line chemotherapy in stage III and IV ovarian cancer. J Clin Oncol. 1997;15:1953-64.

35. Hurria A, Mohile S, Gajra A, Klepin H, Muss H, Chapman A, et al. Validation of a prediction tool for chemotherapy toxicity in older adults with Cancer. J Clin Oncol. 2016;34:2366-71.

Publisher's Note Springer Nature remains neutral with regard to jurisdictional claims in published maps and institutional affiliations. 